\title{
Fibrolamellar Carcinoma
}

National Cancer Institute

\section{Source}

National Cancer Institute. Fibrolamellar Carcinoma. NCI Thesaurus. Code C4131.

A distinctive type of liver cell carcinoma that arises in non-cirrhotic livers and is seen predominantly in young patients. The tumor cells are polygonal and deeply eosinophilic, and are embedded in a fibrous stroma. The prognosis is similar to classical hepatocellular carcinoma that arises in non-cirrhotic livers, and better than hepatocellular carcinoma that arises in cirrhotic livers. 